\title{
Altered presynaptic function and number of mitochondria in the medial prefrontal cortex of adult Cyfip2 heterozygous mice
}

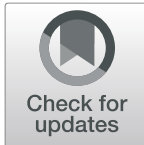

Gyu Hyun Kim ${ }^{1 \dagger}$, Yinhua Zhang ${ }^{2,3+}$, Hyae Rim Kang ${ }^{2,3 \dagger}$, Seung-Hyun Lee ${ }^{4 \dagger}$, Jiwon Shin ${ }^{1}$, Chan Hee Lee ${ }^{1}$, Hyojin Kang ${ }^{5}$, Ruiying Ma ${ }^{2,3}$, Chunmei Jin ${ }^{2,3}$, Yoonhee Kim², Su Yeon Kim², ${ }^{2}$ Seok-Kyu Kwon ${ }^{6}$, Se-Young Choi ${ }^{4^{*}}$, Kea Joo Lee ${ }^{1,7^{*}}$ and Kihoon Han ${ }^{2,3^{*}}$

\begin{abstract}
Variants of the cytoplasmic FMR1-interacting protein (CYFIP) gene family, CYFIP1 and CYFIP2, are associated with numerous neurodevelopmental and neuropsychiatric disorders. According to several studies, CYFIP1 regulates the development and function of both pre- and post-synapses in neurons. Furthermore, various studies have evaluated CYFIP2 functions in the postsynaptic compartment, such as regulating dendritic spine morphology; however, no study has evaluated whether and how CYFIP2 affects presynaptic functions. To address this issue, in this study, we have focused on the presynapses of layer 5 neurons of the medial prefrontal cortex (mPFC) in adult Cyfip2 heterozygous (Cyfip2 ${ }^{+/-}$) mice. Electrophysiological analyses revealed an enhancement in the presynaptic short-term plasticity induced by high-frequency stimuli in Cyfip $2^{+/-}$neurons compared with wild-type neurons. Since presynaptic mitochondria play an important role in buffering presynaptic $\mathrm{Ca}^{2+}$, which is directly associated with the short-term plasticity, we analyzed presynaptic mitochondria using electron microscopic images of the mPFC. Compared with wild-type mice, the number, but not the volume or cristae density, of mitochondria in both presynaptic boutons and axonal processes in the MPFC layer 5 of $\mathrm{Cyfip}^{+/-}$mice was reduced. Consistent with an identification of mitochondrial proteins in a previously established CYFIP2 interactome, CYFIP2 was detected in a biochemically enriched mitochondrial fraction of the mouse mPFC. Collectively, these results suggest roles for CYFI P2 in regulating presynaptic functions, which may involve presynaptic mitochondrial changes.
\end{abstract}

Keywords: Presynapse, Mitochondria, Medial prefrontal cortex, Cytoplasmic FMR1-interacting protein 2

\footnotetext{
*Correspondence: sychoi@snu.ac.kr; relaylee@kbri.re.kr; neurohan@korea.ac.kr

${ }^{\dagger}$ Gyu Hyun Kim, Yinhua Zhang, Hyae Rim Kang and Seung-Hyun Lee contributed equally to this work.

${ }^{4}$ Department of Physiology, Dental Research Institute, Seoul National

University School of Dentistry, 101, Daehak-ro, Jongno-gu, Seoul 03080,

South Korea

${ }^{1}$ Neural Circuits Research Group, Korea Brain Research Institute, 61,

Cheomdan-ro, Dong-gu, Daegu 41062, South Korea

${ }^{2}$ Department of Neuroscience, College of Medicine, Korea University, 73,

Goryeodae-ro, Seongbuk-gu, Seoul 02841, South Korea

Full list of author information is available at the end of the article
}

(C) The Author(s). 2020 Open Access This article is licensed under a Creative Commons Attribution 4.0 International License, which permits use, sharing, adaptation, distribution and reproduction in any medium or format, as long as you give appropriate credit to the original author(s) and the source, provide a link to the Creative Commons licence, and indicate if changes were made. The images or other third party material in this article are included in the article's Creative Commons licence, unless indicated otherwise in a credit line to the material. If material is not included in the article's Creative Commons licence and your intended use is not permitted by statutory regulation or exceeds the permitted use, you will need to obtain permission directly from the copyright holder. To view a copy of this licence, visit http://creativecommons.org/licenses/by/4.0/ The Creative Commons Public Domain Dedication waiver (http://creativecommons.org/publicdomain/zero/1.0/) applies to the data made available in this article, unless otherwise stated in a credit line to the data. 


\section{Main text}

The two members of the cytoplasmic FMR1-interacting protein (CYFIP) family, CYFIP1 and CYFIP2, are evolutionarily highly conserved proteins involved in actin cytoskeleton dynamics and mRNA regulation in neurons [1]. Importantly, both CYFIP1 and CYFIP2 genes are associated with various types of brain disorders, including autism spectrum disorders, intellectual disability, schizophrenia, and epilepsy, suggesting critical roles of CYFIP1 and CYFIP2 in proper brain development and function $[2,3]$. Specifically, in the synaptic compartment, CYFIP1 regulates presynaptic vesicle release [4], excitatory postsynaptic dendritic spine morphology [5], and inhibitory synaptic assembly and transmission [6]. The roles of CYFIP2 in regulating dendritic spine morphology and excitatory synaptic transmission have been investigated [7-9], but its roles in presynaptic functions are largely unknown.

In our previous study, we observed a decrease in the number of presynaptic docked vesicles in layer 5 (L5) neurons in the medial prefrontal cortex (mPFC) of adult Cyfip2 heterozygous (Cyfip $2^{+/}$) mice compared with wild-type mice [8]. Therefore, in this study, we further investigated the presynaptic functional changes in the mPFC L5 neurons of Cyfip $2^{+/-}$mice by measuring the short-term plasticity induced by trains of stimuli [see Additional File 1 for methods]. There was no significant difference between wild-type and Cyfip $2^{+/-}$neurons in terms of the normalized evoked excitatory postsynaptic currents (eEPSCs) induced by a moderate-frequency (10 $\mathrm{Hz}$ ) train of stimuli (Fig. 1a). Both neurons showed similar timings of depression during the train. However, with a high-frequency $(20 \mathrm{~Hz})$ train of stimuli, the normalized eEPSCs were significantly different between wild-type and Cyfip $2^{+/-}$neurons (Fig. 1b). Specifically, Cyfip $2^{+/-}$ neurons showed an overall enhancement in normalized eEPSCs compared with wild-type neurons, suggesting altered short-term plasticity induced by high-frequency stimulation in Cyfip2 $2^{+/-}$neurons.

Presynaptic $\mathrm{Ca}^{2+}$ is critically involved in short-term plasticity [10], and presynaptic mitochondria play an important role in buffering presynaptic $\mathrm{Ca}^{2+}[11,12]$. Notably, a recent study has revealed changes in mitochondrial activity and size in Drosophila Cyfip mutants [13]. Therefore, we also investigated the number and morphology of presynaptic mitochondria in the mPFC L5 neurons of Cyfip $2^{+/-}$mice by re-analyzing electron microscopic image datasets previously acquired for dendritic spine analysis of the MPFC neurons [8] [see Additional File 1 for methods]. We found that mitochondria were observed only in a subpopulation of presynaptic boutons contacting dendritic spines of the mPFC L5 neurons, and that the ratio of presynaptic boutons containing mitochondria in $C y f i p 2^{+/-}$mice was significantly lower than that observed in wild-type mice (Fig. 1c). Considering normal densities of dendritic spines [8] and presynaptic boutons (Fig. 1c) of mPFC L5 neurons in Cyfip $2^{+/-}$mice, these results indicate that Cyfip $2^{+/-}$neurons have more mitochondria-free presynaptic boutons. Additionally, mitochondria number in axonal processes of the mPFC L5 was also significantly decreased in Cyfip2 $2^{+/-}$mice compared with wild-type mice (Fig. 1d), suggesting that mitochondria transport along the axons can also be abnormal in Cyfip $2^{+/-}$mice. In contrast, the mitochondrial volume in neither presynaptic boutons nor axonal processes was altered in Cyfip $2^{+/-}$mice (Fig. 1c,d). As mitochondrial cristae density is highly correlated with its energy production and metabolic capacity [14], we next analyzed the mitochondrial cristae density in Cyfip $2^{+/-}$and wild-type mice. Consistent with no difference in presynaptic mitochondrion volume (Fig. 1c), the cristae density of presynaptic mitochondria was comparable between genotypes (Fig. 1e). These results suggest that Cyfip2 haploinsufficiency affects the axonal and presynaptic localization, rather than the volume or cristae integrity, of mitochondria in the mPFC layer 5.

We have recently identified 140 proteins in the CYFI P2 interactome of mouse forebrain, which mainly included actin-regulatory proteins and RNA-binding proteins [15]. Notably, we found that 23 mitochondrial proteins were also detected in the CYFIP2 interactome (Fig. 1f). To further validate whether CYFIP2 was associated with mitochondria in the MPFC, we biochemically enriched the mitochondrial fraction from the $\mathrm{mPFC}$ homogenate of adult wild-type mice [see Additional File 1 for methods]. Western blot analysis showed that CYFI P2 was detected in the $\mathrm{MPFC}$ mitochondrial fraction together with other mitochondrial proteins (Fig. 1g).

In this study, we showed changes in presynaptic shortterm plasticity in the MPFC L5 neurons of adult Cyfip $2^{+/-}$ mice. The enhancement in the normalized eEPSCs in Cyfip $2^{+/-}$neurons was induced by high-frequency, but not low-frequency, stimuli, suggesting that the decay of presynaptic $\mathrm{Ca}^{2+}$ signaling, such as $\mathrm{Ca}^{2+}$ clearance, rather than the action potential-induced $\mathrm{Ca}^{2+}$ increase, may be altered in Cyfip $2^{+/-}$neurons. Presynaptic mitochondria positively regulate $\mathrm{Ca}^{2+}$ clearance in the axon terminal and thereby modulate neurotransmitter release [12]. Therefore, we speculate that the reduced number of presynaptic mitochondria in Cyfip2 $2^{+/-}$neurons may decrease presynaptic $\mathrm{Ca}^{2+}$ clearance, which in turn elevates the residual $\mathrm{Ca}^{2+}$, and may trigger the short-term facilitating transmission. However, other possible mechanisms should also be considered. For example, mitochondria generate the majority of presynaptic ATP via oxidative phosphorylation, which is critical for synaptic vesicle recycling [11]. Therefore, changes in presynaptic ATP levels owing to the reduced 


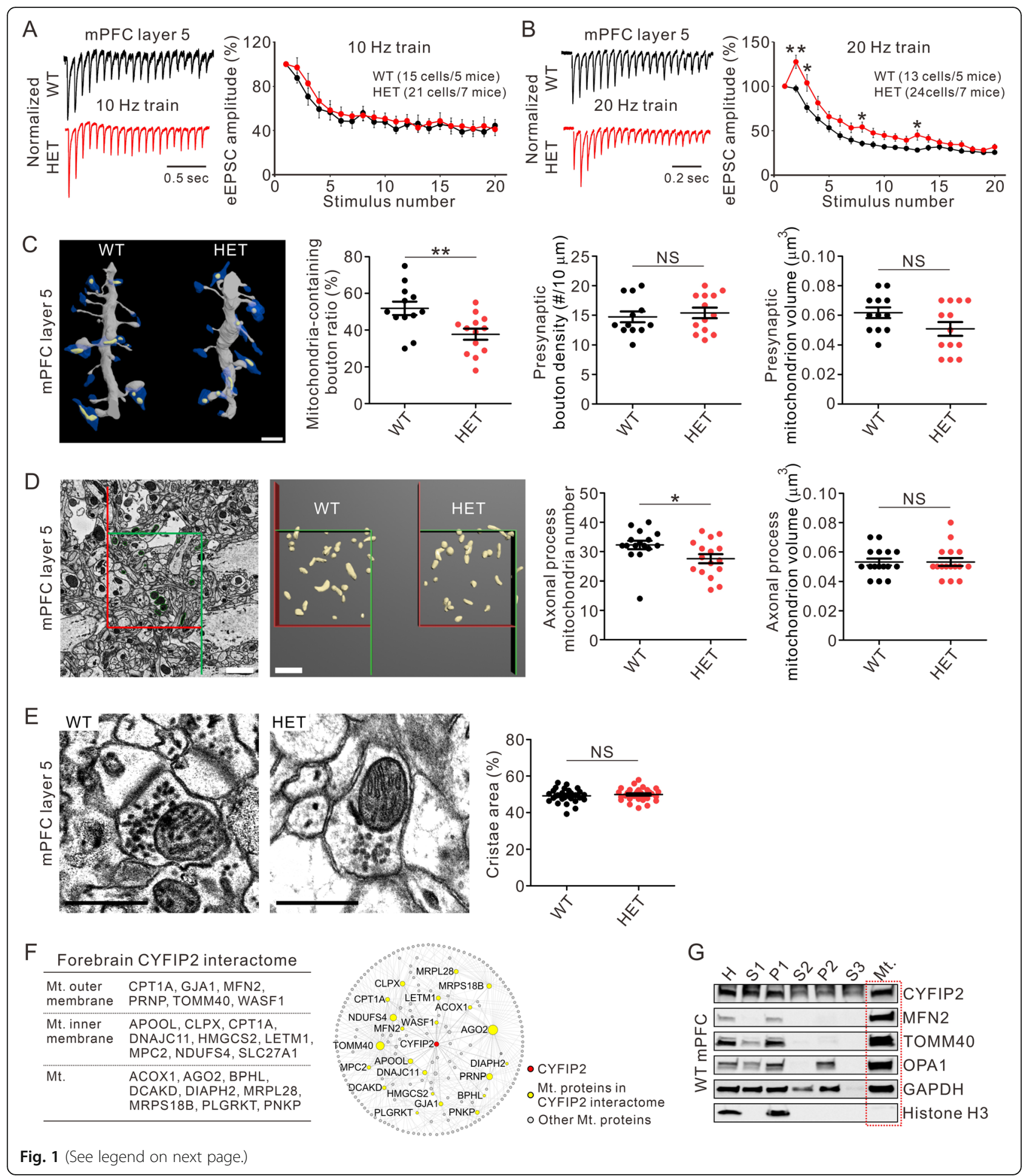


(See figure on previous page.)

Fig. 1 Characterization of presynaptic short-term plasticity and mitochondria in the mPFC of adult Cyfip2 ${ }^{+/-}$mice. a Representative traces and quantification of eEPSCs (normalized to the first eEPSC amplitude) induced by a $10 \mathrm{~Hz}$ train of stimuli in the mPFC layer 5 neurons of wild-type (WT) and $\mathrm{Cyfip}^{+/-}$(heterozygous, HET) mice. Numbers of cells and mice used for the experiments are indicated. $\mathbf{b}$ Representative traces and quantification of normalized eEPSCs induced by a $20 \mathrm{~Hz}$ train of stimuli. c Representative dendritic segments reconstructed from serial block-face scanning electron microscopy (SB-SEM) images show dendritic spines (gray), presynaptic boutons (blue), and presynaptic mitochondria (yellow) in the mPFC layer 5 neurons of WT and HET mice (left panel). Quantification of the mitochondria-containing bouton ratio, presynaptic bouton density, and presynaptic mitochondron volume (right panels, $n=12$ and 13 dendritic segments from 4 WT and 4 HET mice, respectively). Scale bar, $2 \mu \mathrm{m}$. NS, not significant. $\mathbf{d}$ Representative electron microscopic image and reconstruction of axonal process mitochondria in the mPFC layer 5 of WT and HET mice (left panels). Quantification of the number (in $73.5 \mu \mathrm{m}^{3}$ ) and volume of mitochondria (right panels, $n=16$ frames from 4 mice per genotype). Scale bars, $2 \mu \mathrm{m}$. e Representative electron microscopic images of the cristae structures of presynaptic mitochondria in the mPFC layer 5 of WT and HET mice (left panels). Quantification of the mitochondrial cristae density (right panel, $n=30$ mitochondria from 3 mice per genotype). Scale bars, $0.5 \mu \mathrm{m}$. $\mathbf{f}$ List of 23 mitochondrial proteins of the forebrain CYFIP2 interactome (left panel). Interaction network of 23 mitochondrial proteins (yellow nodes) in the CYFIP2 interactome. Other mitochondrial proteins (grey nodes) were also included to generate the network. Mt., mitochondria. g Representative western blot images showing detection of CYFIP2 in mitochondrial fraction from the MPFC homogenate of adult WT mice. Mitofusin 2 (MFN2), translocase of outer mitochondrial membrane 40 (TOMM40), and optic atrophy 1 (OPA1) are representative mitochondrial proteins. Histone $\mathrm{H} 3$ is a nuclear protein (negative control). Data are presented as mean $\pm \mathrm{SEM}$. ${ }^{*} P<0.05$, ${ }^{* *} P<0.01$. (unpaired two-tailed Student's t-test)

mitochondria number may also contribute to the abnormal short-term plasticity in Cyfip $2^{+/-}$neurons. In addition, mitochondria-independent mechanisms, such as changes in presynaptic actin dynamics, can be involved in the presynaptic functional changes of Cyfip $2^{+/-}$neurons, as previously shown in Cyfip1 $1^{+/-}$neurons [4]. Furthermore, in our previous study [8], we showed that the number of docked vesicles in a single presynaptic terminal was lower in Cyfip $2^{+/-}$neurons compared with wild-type neurons, which is also in agreement with our current finding of an enhancement in the presynaptic short-term plasticity and an increase in the pared pulse ratio in Cyfip $2^{+/-}$neurons.

Our observations of reduced number, but normal volume, of presynaptic mitochondria in Cyfip $2^{+/-}$neurons contradict the results of a recent report showing increased mitochondrial activity and size in Drosophila Cyfip mutants [13]. Only one Cyfip gene exists in Drosophila, but there are two genes, Cyfip1 and Cyfip2, in mice. Therefore, one possibility can be that CYFIP1 and CYFIP2 have different roles in regulating presynaptic mitochondria in the mouse brain. Our recent interactome analysis suggested that CYFIP1 and CYFIP2 have distinct pools of binding partners [15], supporting their differential molecular functions in vivo.

Detailed mechanisms underlying the reduced number of mitochondria in presynaptic boutons and axonal processes of Cyfip $2^{+/-}$mPFC require further investigation. Presynaptic mitochondrial transport and localization can be regulated by several mechanisms, including the $\mathrm{Ca}^{2+}$ dependent detachment of the mitochondria from the molecular motor and its immobilization by mitochondrial docking proteins [16]. Whether CYFIP2 is involved in mitochondrial capture at presynaptic sites via interactions with mitochondrial proteins will be an interesting topic of future studies.

Notably, there are several intra-mitochondrial and mitochondrial inner membrane proteins in the CYFIP2 interactome (Fig. 1f). However, we could not find CYFI P2 in the publicly available mitochondrial protein databases (MitoCarta [17] and MitoProteome [18]). Furthermore, intra-mitochondrial localization was not predicted for CYFIP2 by a prediction tool of protein submitochondrial localization (DeepMito [19]). These results suggest that CYFIP2 is not an intra-mitochondrial protein, and that those interactions between CYFIP2 and intra-mitochondrial proteins can be indirect via mitochondrial outer membrane proteins. Further investigations are necessary to identify key mitochondrial proteins mediating CYFIP2-mitochondira interaction and its potential regulation.

In conclusion, our results provide in vivo evidence that CYFIP2 regulates presynaptic functions, which may involve presynaptic mitochondrial changes. These results may be potentially implicated for CYFIP2-associated brain disorders, given that presynaptic mitochondrial dysfunction contributes to the pathogenesis of various brain disorders [11].

\section{Supplementary information}

Supplementary information accompanies this paper at https://doi.org/10. 1186/s13041-020-00668-4.

\section{Additional file 1.}

\section{Abbreviations}

CYFIP: Cytoplasmic FMR1-interacting protein; eEPSC: Evoked excitatory postsynaptic current; HET: Heterozygous; L5: Layer 5; MFN2: Mitofusin 2; mPFC: Medial prefrontal cortex; OPA1: Optic atrophy 1; SB-SEM: Serial blockface scanning electron microscopy; TOMM40: Translocase of outer mitochondrial membrane 40; WT: Wild-type

\section{Acknowledgements}

We thank the Laboratory Animal Research Center at Korea University College of Medicine for their animal care and support, and the Brain Research Core Facilities at Korea Brain Research Institute for electron microscopic support. 


\section{Authors' contributions}

GHK, YZ, HRK, S-HL, JS, CHL, RM, CJ, YK, SYK, and KH designed and performed the experiments. $\mathrm{HK}, \mathrm{S}-\mathrm{KK}, \mathrm{S}-\mathrm{YC}, \mathrm{KJ}$, and $\mathrm{KH}$ analyzed and interpreted the data. S-YC, KJL, and KH wrote the paper. All authors have read and approved the manuscript.

\section{Funding}

This work was supported by the National Research Foundation of Korea (NRF) grants funded by the Korea Government Ministry of Science and ICT (NRF-2018R1C1B6001235, NRF-2018M3C7A1024603, NRF-2017M3C7A1048086, and NRF-2020R1A2C3011464) and the KBRI Basic Research Programs (20-BR01-08 and 20-BR-04-01).

\section{Availability of data and materials}

The datasets used and analyzed in the current study are available from the corresponding authors on reasonable request.

\section{Ethics approval}

The Cyfip $2^{+/-}$mice were bred and maintained in a C57BL/ $6 \mathrm{~J}$ background according to the Korea University College of Medicine Research Requirements. All the experimental procedures were approved by the Committees on Animal Research at the Korea University College of Medicine (KOREA-2018-0174).

\section{Consent for publication}

Not applicable.

\section{Competing interests}

The authors declare that they have no competing interests.

\begin{abstract}
Author details
${ }^{1}$ Neural Circuits Research Group, Korea Brain Research Institute, 61, Cheomdan-ro, Dong-gu, Daegu 41062, South Korea. ${ }^{2}$ Department of Neuroscience, College of Medicine, Korea University, 73, Goryeodae-ro, Seongbuk-gu, Seoul 02841, South Korea. ${ }^{3}$ Department of Biomedical Sciences, College of Medicine, Korea University, Seoul, South Korea. ${ }^{4}$ Department of Physiology, Dental Research Institute, Seoul National University School of Dentistry, 101, Daehak-ro, Jongno-gu, Seoul 03080, South Korea. ${ }^{5}$ Division of National Supercomputing, Korea Institute of Science and Technology Information, Daejeon, South Korea. ${ }^{6}$ Korea Institute of Science and Technology, Center for Functional Connectomics, Brain Science Institute, Seoul 02792, South Korea. ${ }^{7}$ Department of Brain and Cognitive Sciences, DGIST, Daegu, South Korea.
\end{abstract}

Received: 28 July 2020 Accepted: 8 September 2020

Published online: 11 September 2020

\section{References}

1. Zhang $Y$, Lee $Y$, Han K. Neuronal function and dysfunction of CYFIP2: from actin dynamics to early infantile epileptic encephalopathy. BMB Rep. 2019; 52(5):304-11.

2. Abekhoukh S, Bardoni B. CYFIP family proteins between autism and intellectual disability: links with fragile $X$ syndrome. Front Cell Neurosci. 2014:8:81. https://doi.org/10.3389/fncel.2014.00081.

3. Nakashima M, Kato M, Aoto K, Shiina M, Belal H, Mukaida S, et al. De novo hotspot variants in CYFIP2 cause early-onset epileptic encephalopathy. Ann Neurol. 2018;83(4):794-806. https://doi.org/10.1002/ana.25208.

4. Hsiao K, Harony-Nicolas H, Buxbaum JD, Bozdagi-Gunal O, Benson DL. Cyfip1 regulates presynaptic activity during development. J Neurosci. 2016; 36(5):1564-76. https://doi.org/10.1523/JNEUROSCI.0511-15.2016.

5. De Rubeis S, Pasciuto E, Li KW, Fernandez E, Di Marino D, Buzzi A, et al. CYFI P1 coordinates mRNA translation and cytoskeleton remodeling to ensure proper dendritic spine formation. Neuron. 2013;79(6):1169-82. https://doi. org/10.1016/j.neuron.2013.06.039

6. Davenport EC, Szulc BR, Drew J, Taylor J, Morgan T, Higgs NF, et al. Autism and schizophrenia-associated CYFIP1 regulates the balance of synaptic excitation and inhibition. Cell Rep. 2019;26(8):2037-51 e6. https://doi.org/10. 1016/j.celrep.2019.01.092

7. Han K, Chen H, Gennarino VA, Richman R, Lu HC, Zoghbi HY. Fragile X-like behaviors and abnormal cortical dendritic spines in cytoplasmic FMR1- interacting protein 2-mutant mice. Hum Mol Genet. 2015;24(7):1813-23. https://doi.org/10.1093/hmg/ddu595.

8. Lee SH, Zhang Y, Park J, Kim B, Kim Y, Lee SH, et al. Haploinsufficiency of Cyfip2 causes lithium-responsive prefrontal dysfunction. Ann Neurol. 2020. https://doi.org/10.1002/ana.25827.

9. Lee $Y$, Kim D, Ryu JR, Zhang Y, Kim S, Kim Y, et al. Phosphorylation of CYFI $\mathrm{P} 2$, a component of the WAVE-regulatory complex, regulates dendritic spine density and neurite outgrowth in cultured hippocampal neurons potentially by affecting the complex assembly. Neuroreport. 2017;28(12):749-54. https://doi.org/10.1097/WNR.0000000000000838.

10. Regehr WG. Short-term presynaptic plasticity. Cold Spring Harb Perspect Biol. 2012;4(7):a005702. https://doi.org/10.1101/cshperspect.a005702.

11. Devine MJ, Kittler JT. Mitochondria at the neuronal presynapse in health and disease. Nat Rev Neurosci. 2018;19(2):63-80. https://doi.org/10.1038/nrn. 2017.170.

12. Kwon SK, Sando R 3rd, Lewis TL, Hirabayashi Y, Maximov A, Polleux F. LKB1 regulates mitochondria-dependent presynaptic calcium clearance and neurotransmitter release properties at excitatory synapses along cortical axons. PLoS Biol. 2016;14(7):e1002516. https://doi.org/10.1371/journal.pbio. 1002516.

13. Kanellopoulos AK, Mariano V, Spinazzi M, Woo YJ, McLean C, Pech U, et al. Aralar sequesters GABA into hyperactive mitochondria. Causing Social Behavior Deficits Cell. 2020;180(6):1178-97 e20. https://doi.org/10.1016/j.cell. 2020.02.044.

14. Leveille CF, Mikhaeil JS, Turner KD, Silvera S, Wilkinson J, Fajardo VA. Mitochondrial cristae density: a dynamic entity that is critical for energy production and metabolic power in skeletal muscle. J Physiol. 2017;595(9): 2779-80.

15. Lee $Y$, Zhang Y, Kang H, Bang G, Kim Y, Kang HR, et al. Epilepsy-and intellectual disability-associated CYFIP2 interacts with both actin regulators and RNA-binding proteins in the neonatal mouse forebrain. Biochem Biophys Res Commun. 2020;529(1):1-6.

16. Sheng $\mathrm{ZH}$, Cai Q. Mitochondrial transport in neurons: impact on synaptic homeostasis and neurodegeneration. Nat Rev Neurosci. 2012;13(2):77-93. https://doi.org/10.1038/nrn3156.

17. Calvo SE, Clauser KR, Mootha VK. MitoCarta2.0: an updated inventory of mammalian mitochondrial proteins. Nucleic Acids Res. 2016:44(D1):D1251-7. https://doi.org/10.1093/nar/gkv1003.

18. Cotter D, Guda P, Fahy E, Subramaniam S. MitoProteome: mitochondrial protein sequence database and annotation system. Nucleic Acids Res. 2004; 32:D463-D7. https://doi.org/10.1093/nar/gkh048.

19. Savojardo C, Bruciaferri N, Tartari G, Martelli PL, Casadio R. DeepMito: accurate prediction of protein sub-mitochondrial localization using convolutional neural networks. Bioinformatics. 2020;36(1):56-64. https://doi. org/10.1093/bioinformatics/btz512.

\section{Publisher's Note}

Springer Nature remains neutral with regard to jurisdictional claims in published maps and institutional affiliations.

Ready to submit your research? Choose BMC and benefit from:

- fast, convenient online submission

- thorough peer review by experienced researchers in your field

- rapid publication on acceptance

- support for research data, including large and complex data types

- gold Open Access which fosters wider collaboration and increased citations

- maximum visibility for your research: over $100 \mathrm{M}$ website views per year

At $\mathrm{BMC}$, research is always in progress.

Learn more biomedcentral.com/submissions 